\title{
Aspectos limnológicos de estanques para la producción intensiva de tenca (Tinca tinca)
}

\author{
Celia de la Vega ${ }^{1}$, Concha Jambrina ${ }^{2}$, Raúl de $\mathrm{Saja}^{2}$, Eloy Bécares ${ }^{3}$, Camino Fernández $^{3, *}$, \\ Margarita Fernández ${ }^{3}$
}

1 Instituto de Medio Ambiente, Universidad de León, La Serna 56, 24 007, León

2 Tencas de Casaseca, San Lorenzo 1, 47001 Valladolid

3 Área Ecología, Facultad de Biología, Universidad de León, 24071 León

* Autor responsable de la correspondencia: ebecm@unileon.es

\section{RESUMEN}

\section{Aspectos limnológicos de estanques para la producción intensiva de tenca (Tinca tinca)}

La Tenca está considerada una especie de crecimiento lento si se compara con otros ciprínidos, aunque la presencia de zooplancton en sistemas intensivos mejora significativamente el crecimiento de juveniles. Para determinar con mayor exactitud cuales son los factores que influyen en la producción de tenca en condiciones semiintensivas, se llevó a cabo un experimento en 9 estanques de engorde combinando diferentes condiciones de abonado ( 3 estanques control, 3 estanques con abonado inorgánico y 3 estanques con abonado orgánico). Desde julio a septiembre se realizaron muestreos quincenales de todas las características físico-químicas y del zooplancton con el fin de comprobar el efecto de las condiciones experimentales sobre dichas variables. En el estudio realizado se han identificado un total de 57 taxones: 6 copépodos, 18 cladóceros y 33 rotíferos. La abundancia zooplanctónica total fue mayor en los tratamientos fertilizados que en el control, siendo este incremento significativo para el caso del tratamiento inorgánico, lo que indica un efecto positivo del incremento en la carga de nutrientes sobre las comunidades zooplanctónicas. Aunque no se observaron diferencias significativas entre los tratamientos orgánico e inorgánico, la biomasa fue ligeramente superior en el tratamiento inorgánico. La composición faunística en los dos tratamientos fue similar. La comunidad zooplanctónica estuvo, en general, dominada por rotíferos y pequeños cladóceros, siendo los nauplios una fracción muy importante de dicha comunidad.

Palabras clave: Piscicultura, enriquecimiento de nutrients, zooplancton, Tinca tinca, tenca.

\section{ABSTRACT \\ Limonologycal aspects of fishponds for intensive tench (Tinca tinca) production}

Tench is considered a slow growing species in comparison with other ciprinids, even though high zooplancton densities in intensive farms significantly improve juvenile growth. In order to better study the which factors are important on trench production under semi-intensive conditions, an experiment was carried out, in nine triplicates pools combining different fertilizers (natural manure or nitrates) (3 inorganic pools, 3 organic pools and 3 control pools). From July to September 2003, fortnightly samples were taken from physical-chemical and zooplankton to test the effect of the experimental conditions over those variables. From the study, a total of 57 taxa were identified: 6 copepoda, 18 cladocerans and 33 rotifers. Total zooplankton abundance was higher in the fertilized treatments than in the control, the differences being significant in the inorganic treatment, which indicates a positive effect of the increment in the nutrient load over the zooplanktic communities. Even though no significant differences were observed among inorganic and organic fertilizers, the biomass was slightly superior in the inorganic treatment. The faunistic composition in both treatments was similar. The zooplankton community was dominated by rotifers, small cladocerans, and nauplii the latter being an important fraction of the community.

Keywords: Fish production, nutrient enrichment, zooplankton, Tinca tinca, tench. 


\section{INTRODUCCIÓN}

La Tenca (Tinca tinca) está considerada una especie de crecimiento lento si se compara con otros ciprínidos, habiéndose demostrado que en sistemas intensivos la presencia de zooplancton mejora significativamente su crecimiento. El zooplancton constituye la parte más importante en la dieta de los alevines, que se alimentan de manera selectiva, predando principalmente cladóceros y copépodos junto con larvas de insectos cuando van siendo de mayor tamaño (Kennedy \& Fitzmaurice, 1970). Frecuentemente la producción está limitada por la abundancia de las poblaciones zooplanctónicas. Con el objetivo de estimular el desarrollo de comunidades zooplanctónicas, la adicción de abono orgánico en los estanques de engorde de las piscifactorías ha venido siendo una práctica habitual para la cría de peces. Los nutrientes presentes en los fertilizantes se incorporan a la biomasa de fitoplancton y zooplancton y de ahí, a través de la cadena trófica, a la biomasa de los peces. Un incremento en la carga de nutrientes resulta, pues, en una biomasa zooplanctónica elevada y en un alto crecimiento de la producción de peces (Doering et al., 1989; Keller et al., 1990).

Los peces y los nutrientes son considerados como los factores que controlan la biomasa total de zooplancton en los sistemas acuáticos (Carpenter et al., 1987; Persson et al., 1988), pero la importancia relativa de estos controles top-down y bottom-up es aún tema de discusión (McQueen et al., 1986; Jeppesen et al., 1997; 1998, Fernández Aláez et al., 2004)). Mediante manipulación de los niveles tróficos superiores (Carpenter et al., 1987; Horppila et al., 1998, Kairesalo et al., 1999) y de la disponibilidad de nutrientes (Hansson, 1992; Persson et al., 1992; Sarnelle, 1992) se ha demostrado una fuerte estructuración de las cadenas tróficas en los sistemas acuáticos. Sin embargo, las respuestas frente a la manipulación de nutrientes han sido sorprendentemente muy variables (Mazumder, 1994; Brett \& Goldman, 1997; Leibold et al., 1997), lo que en el caso de las prácticas llevadas a cabo en acuicultura se ha relacionado con la influencia de factores como la localización, la fuente morfometría y sedimentos (Knud-Hansen, 1998).

Por otro lado, un excesivo abonado orgánico puede conducir a un deterioro de la calidad de las aguas, causado principalmente por una disminución del oxígeno disuelto y una alta demanda química de oxígeno (Boyd, 1982). Incluso en un agua bien oxigenada, la adición de fertilizantes orgánicos se traduce en un incremento de las enfermedades epidérmicas en los peces (Iger et al., 1988).

Para determinar con mayor exactitud cuales son los factores que influyen de forma significativa en la productividad natural y su influencia en el crecimiento, es fundamental establecer un programa de muestreo regular que permita evaluar las características de calidad de agua en los estanques, las comunidades que afloran en los mismos y el aprovechamiento real que los peces hacen de este recurso natural en el proceso de engorde. Así, como base para un estudio comparativo, los datos obtenidos en estanques "control" son necesarios para determinar el posible impacto ambiental y las mejoras que en el sistema de cría pueden derivarse del empleo de métodos de abonado (orgánico e inorgánico) orientados a inducir la productividad natural. El objetivo de este estudio ha sido estudiar desde el punto de vista cualitativo (especies y diversidad) y cuantitativo (abundancia y biomasa) las comunidades zooplanctónicas que se desarrollan en los estanques, tratando de evaluar su respuesta en función del fertilizante añadido, orgánico e inorgánico y además contribuir al conocimiento de la biología de la tenca en condiciones naturales con el fin de ejecutar medidas de recuperación ecológica en lagunas biológicamente deterioradas.

\section{ÁREA DE ESTUDIO}

Los estanques elegidos para el estudio se ubican en las instalaciones de la empresa "Tencas de Casaseca S.L" situada en el término municipal de Casaseca de las Chanas, en la provincia de Zamora. La piscifactoría está situada en una zona con clima mediterráneo templado, siendo la temperatura media anual de $13.2{ }^{\circ} \mathrm{C}$ 
y la precipitación media anual de $443 \mathrm{~mm}$. El agua está presente en la zona de dos formas: aguas superficiales del arroyo de Ariballos, que vierte directamente en el río Duero; y aguas subterráneas que afloran a la superficie en numerosos puntos.

Para el desarrollo de este proyecto se ha contado con 9 estanques de $1500 \mathrm{~m}^{2}$ con una profundidad media de $1.60 \mathrm{~m}$, construidos sobre el medio natural, limitándose el uso de hormigón a los taludes de los estanques para evitar la presencia de plantas invasoras no deseadas que dificultan el cultivo de la tenca.

\section{METODOLOGÍA}

A finales de febrero y comienzos de Marzo del 2003, se llenaron con agua procedente de un manantial subterráneo 9 tanques de engorde, en los que se aplicaron diferentes condiciones de abonado: Tres tanques se abonaron con 320 kilos de estiércol de vaca, tres con $9 \mathrm{~kg}$ de nitrato amónico $+3 \mathrm{~kg}$ de superfosfato $+3 \mathrm{~kg}$ de cloruro potásico y otros tres estanques no recibieron abonado, sirviendo como control. En todos ellos se añadieron alevines de tenca de un peso inferior a los 50 gr. (la mayoría no pasaban de 5 gr) y en número de entre 12000 y 15000 individuos por tanque.

La tipificación de los estanques de engorde desde el punto de vista físico-químico y biológico se llevó a cabo mediante la recogida de muestras de agua con una periodicidad quincenal durante los meses de julio y agosto del año 2003. In situ se midieron en el agua las siguientes variables: temperatura, $\mathrm{pH}$, conductividad y oxígeno disuelto, utilizando un sistema de medida GRANT/YSI "Water Quality Logger 3800”. Se efectuó además la recogida de muestras de agua para la determinación en el laboratorio de nitrato, amonio, fósforo total, ortofosfato y clorofila $a$. En la recogida y conservación de las muestras y en la determinación analítica se siguió la metodología de Standard Methods, APHA (1985).

Se procedió también a la recogida de zooplancton, filtrando una cantidad conocida de agua, 5 litros para crustáceos y 3 litros para rotíferos a través de Nytal de $50 \mu \mathrm{m}$ y $25 \mu \mathrm{m}$ de luz de malla respectivamente. Una vez filtrada el agua se procedió a la fijación de la muestra, añadiendo una bebida carbónica como narcótico para evitar la contracción del tegumento de los organismos, preservando despues la muestra con formaldehído al $4 \%$. En la determinación taxonómica se utilizaron las claves de Alonso (1996) para la identificación de cladóceros, las de Braioni \& Gelmini, (1983) para rotíferos y las de Dussart, $(1967,1969)$ para copépodos. Se obtuvieron datos de la densidad (ind/l) y biomasa $(\mu \mathrm{g} / \mathrm{l})$ de cada uno de los taxones presentes en los estanques. El cálculo de la biomasa se realizó multiplicando el número de individuos de cada una de los taxones por el peso seco obtenido a

Tabla 1. Valores medios de las variables físicas y químicas y resultados del ANOVA de medidas repetidas (nivel de significación) para cada uno de los parámetros estudiados. (Trat: Tratamiento; Tiempo; $\operatorname{Tr} \times \mathrm{T}$ : Interacción Tiempo-tratamiento; C: Control; I: Inorgánico; O: Orgánico). Mean values of the physico-chemical variables and results ( $P$-values) from the repeated measures ANOVA test for each one the studied parameters. (Trat: treatment, Tiempo: time, $\operatorname{Tr} \times$ T: interaction; C: control tanks, I: inorganic fertilizer, O: organic fertilizer)

\begin{tabular}{|c|c|c|c|c|c|c|c|c|c|c|c|c|c|c|c|c|}
\hline & \multicolumn{2}{|c|}{$\begin{array}{c}\text { Nitrato } \\
m g / L\end{array}$} & \multicolumn{2}{|c|}{$\begin{array}{c}\text { Amonio } \\
\mu g / L\end{array}$} & \multicolumn{2}{|c|}{$\begin{array}{c}\text { Fósforo } T \\
\quad \mu g / L\end{array}$} & \multicolumn{2}{|c|}{$\begin{array}{c}\text { Ortofosfato } \\
\mu \mathrm{g} / \mathrm{L}\end{array}$} & \multicolumn{2}{|c|}{$\begin{array}{c}\text { Clorofila a } \\
\quad \mu g / L\end{array}$} & \multicolumn{2}{|c|}{$\begin{array}{c}\mathrm{O}_{2} \\
m g / L\end{array}$} & \multicolumn{2}{|c|}{$p H$} & \multicolumn{2}{|c|}{$\begin{array}{c}\text { Conductividad } \\
\mu \mathrm{S} / \mathrm{cm}\end{array}$} \\
\hline & $X$ & $D E$ & $X$ & $D E$ & $X$ & $D E$ & $X$ & $D E$ & $X$ & $D E$ & $X$ & $D E$ & $X$ & $D E$ & $X$ & $D E$ \\
\hline$C$ & 0.14 & 0.14 & 13.40 & 16.0 & 92.59 & 27.7 & 9.55 & 5.7 & 14.5 & 10.4 & 6.5 & 1.2 & 8.5 & 0.6 & 464 & 57 \\
\hline$I$ & 0.13 & 0.13 & 10.01 & 11.8 & 176.58 & 30.7 & 25.28 & 8.5 & 33.4 & 7.5 & 7.9 & 1.5 & 8.7 & 1.0 & 435 & 48 \\
\hline$O$ & 0.16 & 0.12 & 27.70 & 31.4 & 166.57 & 37.5 & 18.93 & 5.0 & 34.3 & 9.9 & 8.6 & 1.0 & 8.7 & 0.7 & 452 & 42 \\
\hline Trat & \multicolumn{2}{|c|}{0.63} & \multicolumn{2}{|c|}{0.14} & \multicolumn{2}{|c|}{0.05} & \multicolumn{2}{|c|}{0.03} & \multicolumn{2}{|c|}{0.05} & \multicolumn{2}{|c|}{0.06} & \multicolumn{2}{|c|}{0.60} & \multicolumn{2}{|c|}{0.74} \\
\hline Tiempo & \multicolumn{2}{|c|}{0.0003} & \multicolumn{2}{|c|}{0.0007} & \multicolumn{2}{|c|}{0.02} & \multicolumn{2}{|c|}{0.006} & \multicolumn{2}{|c|}{0.025} & \multicolumn{2}{|c|}{0.04} & \multicolumn{2}{|c|}{0.00000} & \multicolumn{2}{|c|}{0.000001} \\
\hline$T r \times T$ & \multicolumn{2}{|c|}{0.67} & \multicolumn{2}{|c|}{0.53} & \multicolumn{2}{|c|}{0.73} & \multicolumn{2}{|c|}{0.70} & \multicolumn{2}{|c|}{0.11} & \multicolumn{2}{|c|}{0.69} & \multicolumn{2}{|c|}{0.39} & \multicolumn{2}{|c|}{0.96} \\
\hline
\end{tabular}



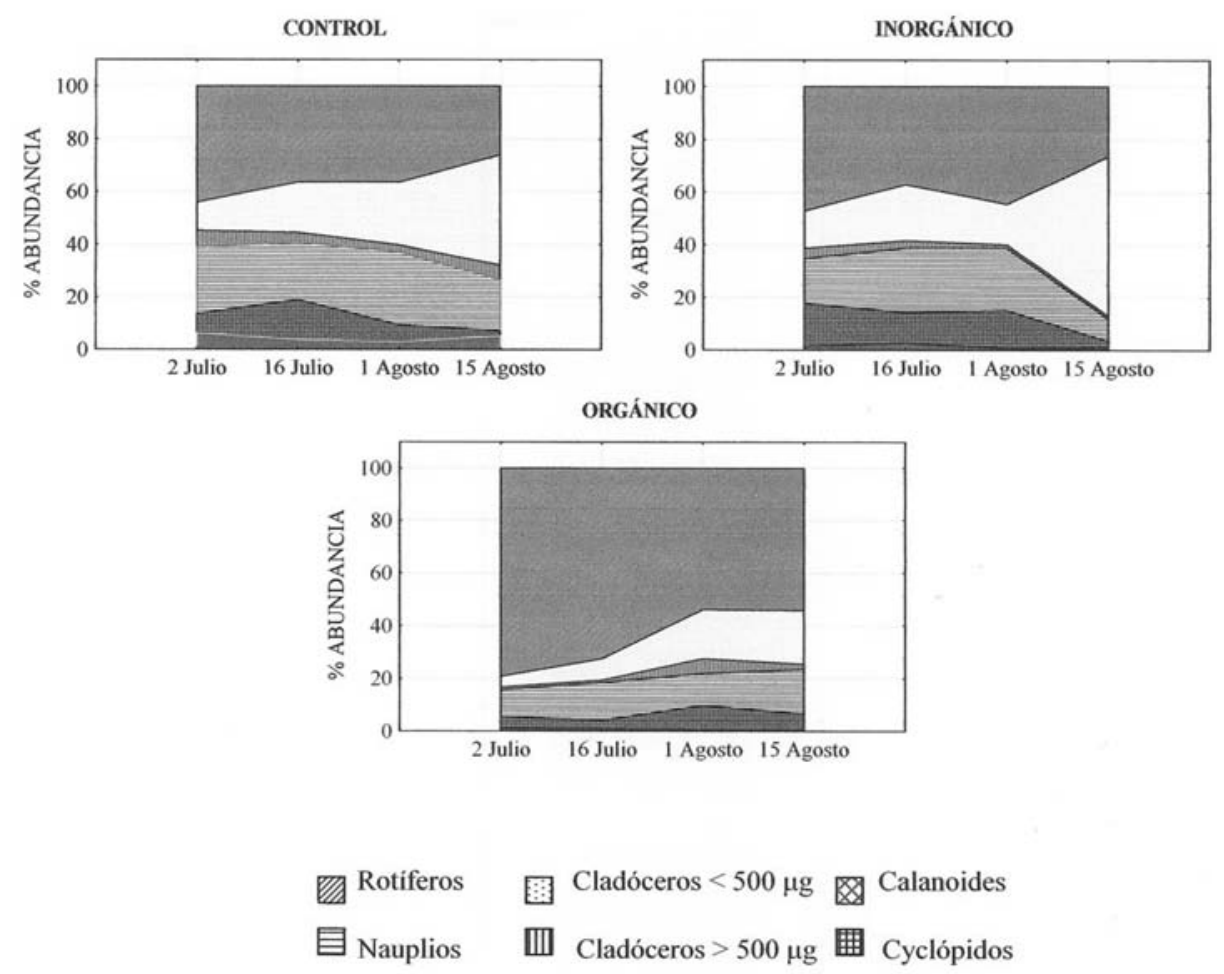

Figura 1. Evolución temporal de la abundancia relativa de los diferentes grupos zooplanctónicos en cada tratamiento (C: Control; I: Inorgánico; O: Orgánico). Temporal variation of the relative abundance for each of the zooplankton groups in each treatment $(C$ : Control; I: Inorganic; O: Organic).

partir de la medida de su longitud y de las ecuaciones de regresión propuestas por Dumont et al., (1975) y por Bottrell et al., (1976).

Se calculó la diversidad de la comunidad zooplanctónica de cada estanque usando el índice de diversidad de Shannon-Weaver y se utilizó el método gráfico tipo box plot para evaluar la respuesta de las diferentes variables medidas frente a los tratamientos aplicados. Mediante ANOVA de medidas repetidas se analizaron las diferencias de las variables químicas, así como de los valores de abundancia y biomasa zooplanctónicas entre tratamientos, comprobando previamente la normalidad de las mismas y la homogeneidad de varianzas mediante los tests de KolmogorovSmirnov y Levene respectivamente Aquellas variables que no cumplieron estas condiciones se transformaron mediante la expresión log $(x+0.1)$. Las variables en las que se observaron diferencias significativas entre los diferentes tratamien- tos fueron analizadas con el test de Tukey para comprobar las diferencias entre pares $(p<0.05)$.

\section{RESULTADOS Y DISCUSIÓN}

\section{Variables físicas y químicas}

En la tabla 1 se recogen los valores medios de las variables físicas y químicas para el periodo de estudio, así como los resultados obtenidos en el ANOVA.

Sólo la concentración de ortofosfato fue significativamente diferente entre tratamientos $(p=0.03)$, si bien estas diferencias afectaron únicamente al tratamiento inorgánico en el que el contenido de este nutriente fue superior al del control. Las restantes variables químicas no mostraron diferencias significativas entre tratamientos, aunque en el caso del fósforo total 
Tabla 2. Listado taxonómico y abundancias medias registradas durante el estudio para cada una de las condiciones experimentales. List showing taxa composition and their mean abundances registered during the study for each of the experimental conditions.

\begin{tabular}{|c|c|c|c|}
\hline & Control & Inorgánico & Orgánico \\
\hline \multicolumn{4}{|l|}{ Copépodos } \\
\hline Hemidiaptomus sp & 12.98 & 15.72 & 6.98 \\
\hline Acanthocyclops capillatus & 3.12 & 10.88 & 4.46 \\
\hline Acanthocyclops robustus & 5.49 & 15.84 & 11.45 \\
\hline Macrocyclops albidus & 4.35 & 0.67 & 0.06 \\
\hline Mesocyclops sp & 0.00 & 0.02 & 0.00 \\
\hline Termocyclops sp & 0.00 & 0.00 & 0.00 \\
\hline Copepoditos Calanoides & 19.35 & 15.27 & 8.93 \\
\hline Copepoditos Ciclopoides & 44.67 & 161.23 & 98.70 \\
\hline Nauplios & 183.80 & 386.28 & 266.84 \\
\hline \multicolumn{4}{|l|}{ Cladóceros } \\
\hline Alona rectangula & 0.30 & 1.75 & 0.94 \\
\hline Alonella nana & 0.11 & 4.47 & 0.09 \\
\hline Bosmina longirostris & 201.73 & 1208.49 & 230.00 \\
\hline Ceriodaphnia quadrangula & 5.08 & 11.08 & 8.84 \\
\hline Ceriodaphnia sp. & 0.00 & 0.00 & 1.19 \\
\hline Chydorus sphaericus & 4.17 & 23.15 & 24.07 \\
\hline Daphnia magna & 0.60 & 0.00 & 14.71 \\
\hline Daphnia longiespina & 2.03 & 22.07 & 11.85 \\
\hline Daphnia pulicaria & 0.02 & 0.00 & 0.00 \\
\hline Dunhevedia crassa & 0.00 & 0.07 & 0.00 \\
\hline Moina sp. & 0.51 & 0.21 & 9.32 \\
\hline Macrothrix hirsuticornis & 0.00 & 0.00 & 0.65 \\
\hline Pleuroxus aduncus & 0.68 & 1.05 & 0.84 \\
\hline Simocephalus vetulus & 0.02 & 2.73 & 0.55 \\
\hline Simocephalus expinosus & 0.10 & 2.44 & 0.03 \\
\hline Diaphanosoma brachyura & 29.34 & 12.81 & 10.06 \\
\hline Ostrácodos & 2.22 & 2.79 & 3.02 \\
\hline \multicolumn{4}{|l|}{ Rotíferos } \\
\hline Anuraeopsis fissa & 0.00 & 0.00 & 0.00 \\
\hline Asphanchna siëboldi & 0.00 & 0.00 & 0.21 \\
\hline Ascomorpha sp & 0.00 & 0.26 & 0.00 \\
\hline Bdelloidea & 2.91 & 2.00 & 7.19 \\
\hline Brachionus angularis & 2.46 & 320.92 & 165.59 \\
\hline Brachionus calyciflorus & 0.00 & 0.00 & 0.00 \\
\hline Brachionus quadridentatus & 0.60 & 3.14 & 0.89 \\
\hline Brachionus patulus & 27.20 & 23.09 & 35.70 \\
\hline Cephalodella sp. & 0.99 & 0.10 & 0.00 \\
\hline Cephalodella gibba & 0.00 & 0.26 & 0.00 \\
\hline Colurella colurus & 0.00 & 0.48 & 0.20 \\
\hline Dicranophoridae (Fam.) & 0.00 & 0.20 & 0.00 \\
\hline Euchlanis dilata & 1.19 & 0.55 & 3.88 \\
\hline Filina longiseta & 1.10 & 18.08 & 100.30 \\
\hline Hexarthra mira & 9.11 & 3.28 & 26.13 \\
\hline Keratella cochclearis & 102.99 & 162.44 & 619.47 \\
\hline Keratella quadrata & 73.23 & 227.90 & 232.21 \\
\hline Lecane bulla & 7.90 & 12.87 & 5.29 \\
\hline
\end{tabular}

(Continúa)

\begin{tabular}{|c|c|c|c|}
\hline & Control & corgánico & Orgánico \\
\hline Lecane closterocerca & 5.14 & 69.64 & 9.15 \\
\hline Lecane luna & 4.27 & 16.58 & 13.05 \\
\hline Lecane lunaris & 0.00 & 0.48 & 0.00 \\
\hline Lecane sp. & 0.00 & 0.30 & 0.00 \\
\hline Lepadella patella & 2.36 & 1.74 & 0.41 \\
\hline Lepadella rhomboides & 3.17 & 2.50 & 3.27 \\
\hline Lophocaris sp & 0.75 & 0.00 & 0.00 \\
\hline Mytilina ventralis & 0.84 & 0.37 & 0.99 \\
\hline Platyas quadricornis & 0.78 & 0.95 & 0.00 \\
\hline Polyarthra vulgaris & 0.81 & 26.30 & 9.03 \\
\hline Squatinella $s p$ & 0.00 & 0.00 & 0.00 \\
\hline Synchaeta g. stylata-pectinata & 2.33 & 0.00 & 4.75 \\
\hline Testudinella patina & 11.55 & 14.95 & 4.00 \\
\hline Trichocerca spp. & 1.87 & 1.53 & 6.02 \\
\hline Trichotria tetractis & 2.42 & 1.19 & 3.87 \\
\hline Trichotria pocillum & 0.24 & 0.30 & 0.00 \\
\hline
\end{tabular}

y la clorofila se observó un incremento en los estanques fertilizados respecto al control.

El ANOVA reveló cambios significativos a lo largo del tiempo (Tabla 1), con una tendencia a disminuir la concentración de todos los nutrientes, clorofila a, pH y oxígeno. Sólo la conductividad aumentó de forma significativa a lo largo de todo el experimento.

\section{Zooplancton}

Se identificaron un total de 57 taxones, de los cuales 6 pertenecieron a copépodos, 18 a cladóceros y 33 a rotíferos, además de copepoditos calanoides y ciclópidos y nauplios. En la tabla 2 se muestra un listado taxonómico con los valores medios de abundancia registrados para cada taxón.

En la figura 1 se muestra la evolución de la abundancia relativa de los diferentes grupos a lo largo del periodo de estudio en los tanques control y en los sometidos a tratamientos orgánico e inorgánico

Los tanques control e inorgánico se asemejaron más entre sí en la abundancia relativa de los diferentes grupos zooplanctónicos, diferenciándose del tratamiento orgánico por un menor predominio de rotíferos y mayor proporción de cladóceros $<500 \mu \mathrm{m}$, especialmente al final del periodo de estudio (Fig. 1). Se observó, así mismo, una mayor proporción de calanoides en el control que en los tanques sometidos a trata- 
Tabla 3. Valores medios de los parámetros estructurales de la comunidad y resultados obtenidos en el ANOVA de medidas repetidas (nivel de significación). (Trat: Tratamiento; Tiempo; $\operatorname{Tr} \times \mathrm{T}$ : Interacción Tiempo-tratamiento; C: Control; I: Inorgánico; O: Orgánico). Mean values of the structural parameters of the community and obtained results using repeated measures ANOVA test ( $P$ values). (Trat: treatment, Tiempo: time, $\operatorname{Tr} \times T$ : interaction; $C$ : control tanks, I: inorganic fertilizer, $O$ : organic fertilizer)

\begin{tabular}{lcccc}
\hline & $\begin{array}{c}\text { Riqueza } \\
\boldsymbol{n}^{\boldsymbol{o}} \text { de taxones }\end{array}$ & $\begin{array}{c}\text { Diversidad } \\
\text { Bits/Ind }\end{array}$ & $\begin{array}{c}\text { Abundancia } \\
\text { Ind/L }\end{array}$ & $\begin{array}{c}\text { Biomasa } \\
\boldsymbol{\mu} \mathbf{g} / \boldsymbol{L}\end{array}$ \\
\hline $\boldsymbol{C}$ & 20 & 2.98 & 787 & 662 \\
$\boldsymbol{I}$ & 21 & 2.64 & 2811 & 1564 \\
$\boldsymbol{O}$ & 20 & 2.82 & 1965 & 1064 \\
\hline Trat. & 0.88 & 0.63 & $\mathbf{0 . 0 4}$ & 0.15 \\
\hline Tiempo & $\mathbf{0 . 0 0 0 0 3}$ & $\mathbf{0 . 0 0 3}$ & $\mathbf{0 . 0 0 9}$ & $\mathbf{0 . 0 0 0 1}$ \\
\hline $\boldsymbol{T r} \times \boldsymbol{T}$ & 0.84 & 0.06 & 0.33 & 0.02 \\
\hline
\end{tabular}

miento. La comunidad zooplanctónica del tratamiento orgánico estuvo dominada claramente por rotíferos (Fig. 1), especies adaptadas a condiciones más eutróficas. Haciendo referencia a esta observación, determinados estudios han demostrado que los nutrientes, conductividad y clorofila determinan la presencia de ciertas especies de rotíferos (Berzins \& Pejler, 1987, 1989; Devetter, 1998). La mayoría de las especies de rotíferos planctónicos filtran o se alimentan de algas y bacterias en suspensión y probablemente son indicadores sensibles de variaciones en el tipo y densidad de alimento causados por cambios en el nivel de nutrientes (Duggan et al., 2001). Varios autores han constatado que el estado trófico afecta decisivamente a la distribución de las especies de rotíferos en los lagos (Siegfried et al., 1989;
Ejsmont-Karabin, 1995; Kaushik \& Saksena, 1995) y a su densidad (Arora \& Mehra, 2003).

Bosmina longirostris fue la especie más abundante en los tanques control e inorgánico, con una media de 201 y 1208 ind/l respectivamente. Se trata de un cladócero euplanctónico frecuente en la zona litoral de los lagos, y asociado a los ambientes eutróficos y poco mineralizados (Armengol, 1978; Alonso, 1985; Margaritora, 1985). En el tratamiento orgánico la mayor densidad correspondió a Keratella cochlearis, con 619 ind/l, estando esta especie también muy representada en los demás tratamientos. Otras especies también muy abundantes en todos los tanques fueron Keratella quadratta, Brachionus angularis, Hexarthra mira, Brachionus patulus, Filinia longiseta, rotíferos frecuentes en aguas con alta carga en nutrientes, siendo alguna de ellas como Keratella quadratta y Brachionus angularis indicadoras de eutrofía (Hutchinson, 1967; Braioni \& Gelmini, 1983). Los copepoditos ciclópidos fueron más abundantes en los tanques inorgánico y orgánico, con abundancias medias de 161 y 99 ind/l respectivamente

La riqueza taxonómica, no mostró diferencias significativas entre tratamientos, con un valor medio en torno a los 20 taxones en todos ellos (Tabla 3). Tampoco la diversidad presentó diferencias entre tratamientos, aunque fue algo menor en los tanques fertilizados y en particular en el inorgánico $\mathrm{y}$, al igual que la riqueza, disminuyó a lo largo del periodo de estudio.

La abundancia zooplanctónica cambió de forma significativa $(p=0.04)$ con la aplicación

Tabla 4. Valores medios de la abundancia y biomasa de cada uno de los grupos estudiados y niveles de significación obtenidos en el ANOVA de medidas repetidas. (Trat: Tratamiento; Tiempo; Tr $\times$ T: Interacción Tiempo-tratamiento; C: Control; I: Inorgánico; O: Orgánico). Mean abundance and biomass values for each of the studied groups, and significant $P$ values obtained in the repeated measures ANOVA. (Trat: treatment, Tiempo: time, $\operatorname{Tr} \times$ T: interaction; $C$ : control tanks, I: inorganic fertilizer, O: organic fertilizer).

\begin{tabular}{|c|c|c|c|c|c|c|c|c|c|c|c|c|}
\hline & \multicolumn{2}{|c|}{ Calanoides } & \multicolumn{2}{|c|}{ Cyclopoides } & \multicolumn{2}{|c|}{ Nauplios } & \multicolumn{2}{|c|}{ Cladóceros < $500 \mu \mathrm{m}$} & \multicolumn{2}{|c|}{ Cladóceros > $500 \mu \mathrm{m}$} & \multicolumn{2}{|c|}{ Rotíferos } \\
\hline & Ind/L & $\mu g / L$ & Ind/L & $\mu g / L$ & Ind/L & $\mu g / L$ & Ind/L & $\mu g / L$ & Ind/L & $\mu g / L$ & Ind/L & $\mu g / L$ \\
\hline$C$ & 32 & 210 & 58 & 142 & 184 & 55 & 211 & 114 & 33 & 74 & 266 & 67 \\
\hline$I$ & 30 & 23 & 189 & 281 & 386 & 80 & 1249 & 641 & 41 & 180 & 912 & 159 \\
\hline$O$ & 15 & 120 & 115 & 152 & 266 & 56 & 265 & 125 & 48 & 423 & 1251 & 188 \\
\hline Trat. & 0.21 & 0.31 & 0.19 & 0.24 & 0.20 & 0.76 & 0.016 & 0.010 & 0.72 & 0.71 & 0.047 & 0.15 \\
\hline Tiempo & 0.02 & 0.04 & 0.02 & 0.03 & 0.03 & 0.19 & 0.00007 & 0.0007 & 0.13 & 0.88 & 0.55 & 0.53 \\
\hline$T r \times T$ & 0.22 & 0.11 & 0.32 & 0.59 & 0.99 & 0.98 & 0.0001 & 0.0013 & 0.11 & 0.22 & 0.89 & 0.99 \\
\hline
\end{tabular}


de los tratamientos (Fig. 2), siendo más elevada en aquellos en los que se utilizó fertilización inorgánica que en los tanques control ( $p=0.04)$, lo que indica un efecto positivo del incremento en la carga de nutrientes sobre las comunidades zooplanctónicas, como ya indicó Fulton (1984). A este aumento contribuyeron fundamentalmente los pequeños cladóceros, $(p=0.016)$ cuya densidad se incremento considerablemente en los tratamientos inorgánicos al final del periodo de estudio debido al extraordinario desarrollo de la población de Bosmina longirostris, que alcanzó densidades superiores a $4200 \mathrm{ind} / \mathrm{l}$. Igualmente, la abundancia de rotíferos aumentó de forma significativa $(p=0.04)$, si bien este aumento se pro- dujo sólo con la fertilización orgánica y no con el tratamiento inorgánico (Fig. 2). Por tanto, sólo en rotíferos y pequeños cladóceros se pueden señalar evidencias directas de que un enriquecimiento diferencial de nutrientes ha modificado la estructura de la comunidad zooplanctónica. En el resto de los grupos, es decir, cladóceros $>500 \mathrm{~mm}$, y copépodos, no se detectaron diferencias significativas en su abundancia por efecto de los tratamientos y sólo la densidad de calanoides disminuyó en el tratamiento orgánico, aunque este cambio tampoco fue significativo.

La abundancia total y la de todos los grupos, a excepción de los grandes cladóceros y los rotíferos, se modificó significativamente a lo
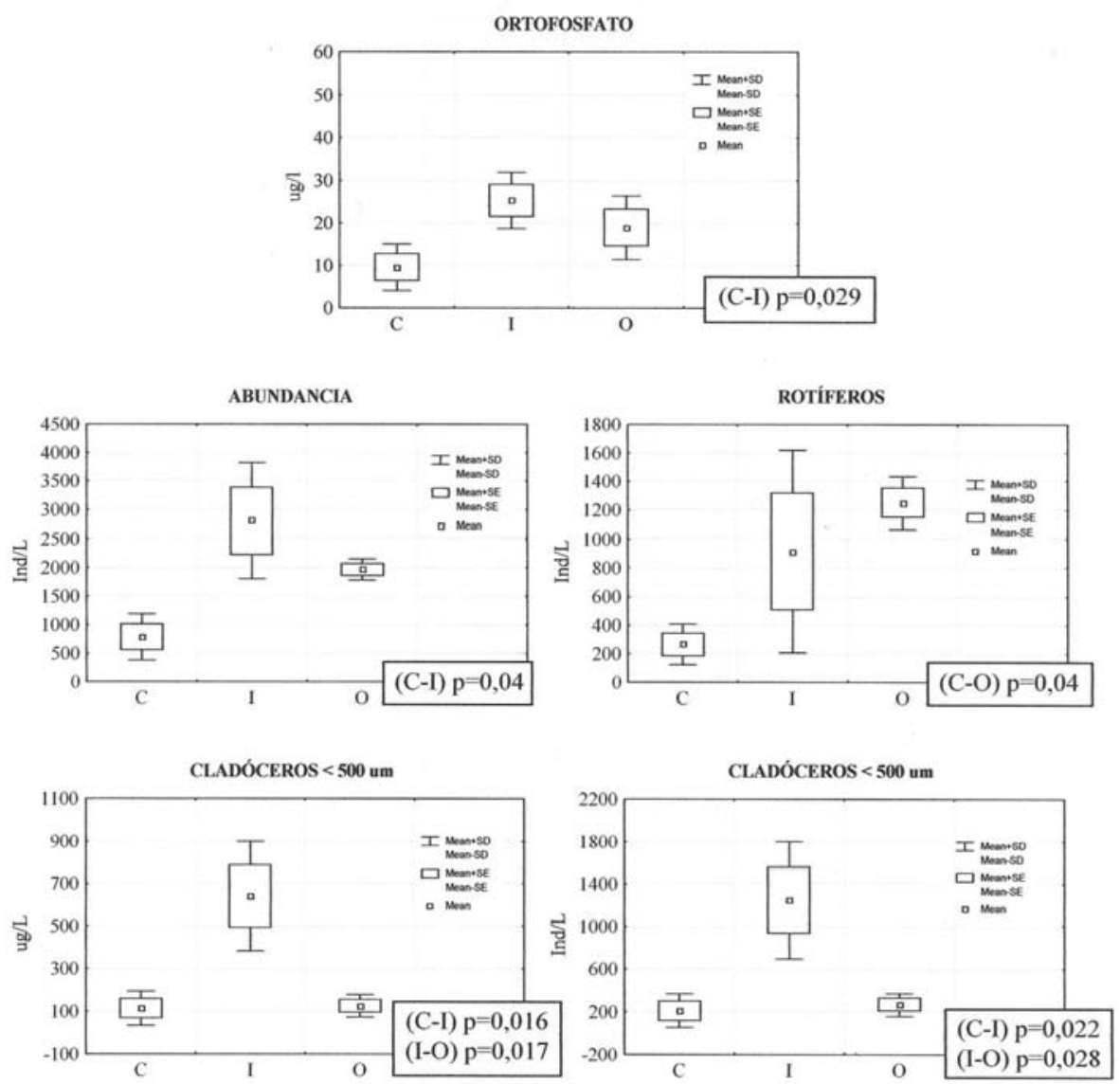

Figura 2. Comparación entre tratamientos para aquellas variables que mostraron diferencias estadísticamente significativas con el ANOVA. Se indican las diferencias entre pares obtenidas mediante el test de Tukey. (C: Control; I: Inorgánico; O: Orgánico). Comparison between treatments for those variables showing statistically significant differences after the ANOVA.. Differences among pairs obtained using Tukey test are shown. (C: control tank, I: inorganic fertilizer, O: organic fertilizer) 
largo del experimento (Tabla 3). En todos los casos, salvo para los ciclópidos, se produjeron aumentos de las poblaciones, que fueron más evidentes en los dos últimos muestreos

En cuanto a la biomasa zooplanctónica, los valores más altos se registraron en los estanques fertilizados, especialmente en el caso de los inorgánicos, si bien las diferencias entre tratamientos no fueron significativas (Tabla 3). Al avanzar el experimento se produjo un aumento significativo de la biomasa total $(p=0.0001)$ en los tanques fertilizados, que afectó sobre todo al tratamiento inorgánico. El aumento de biomasa en los tanques "inorgánicos" se debió, al igual que en el caso de la abundancia, al importante desarrollo de las poblaciones de pequeños cladóceros, que fue el único grupo que mostró una respuesta significativa frente al tratamiento ( $p=0.01)$, sobre todo en la fase final del experimento ( $p=0.0013)$ (Fig. 2). Aunque no fue significativo, el tratamiento inorgánico provocó también un aumento de la biomasa de ciclópidos. No ocurrió así con los calanoides, siendo la biomasa de los estanques control superior a los tratamientos fertilizados, en especial con respecto al tratamiento orgánico, lo que pone de manifiesto la respuesta negativa de este grupo a elevados niveles de nutrientes, observada igualmente por Patalas (1972) (Tabla 4).

El avance del experimento supuso sólo un aumento significativo de la biomasa de pequeños cladóceros $(p=0.0007)$ y de calanoides $(p=0.03)$, mientras que la biomasa de ciclópidos disminuyó ( $p=0.03$ ), particularmente en la última semana. Steiner \& Roy (2003) registraron, igualmente, una respuesta positiva de la biomasa de comunidades zooplanctónicas frente al enriquecimiento en nutrientes, en especial en el caso de pequeños cladóceros como Bosmina longirostris, aceptada como una especie indicadora de eutrofia (Hutchinson, 1967), y característica de aguas muy productivas. Igualmente, Chakrabarti y Jana (1992) constataron un dominio de copépodos como consecuencia de la fertilización y Okonji \& Obi (1999) encontraron que mientras que los fertilizantes orgánicos producían un aumento en el zooplancton de menor tamaño (rotíferos), los inorgánicos favorecían la producción de zooplancton de mayor tamaño (copépodos), lo que resulta importante en el engorde de alevines.

\section{CONCLUSIONES}

Tanto la fertilización orgánica como la inorgánica parecen aportar suficientes nutrientes para asegurar la producción primaria y la producción de peces. Mientras que el tratamiento orgánico parece favorecer más a grupos como los rotíferos, el inorgánico lo hace con grupos como los cladóceros menores de $500 \mu \mathrm{m}$ y, en menor medida, ciclópidos.

Además de la carga de nutrientes otras variables, como los cambios en la cobertura de macrófitos o la extracción de peces de los tanques pueden estar actuando sobre las comunidades zooplanctónicas, lo que explicaría la importante variabilidad encontrada entre estanques sometidos a un mismo tratamiento. Esto dificultaría el establecimiento de una relación válida de causa-efecto.

La producción media de tenca para los estanques control fue de $642.5 \mathrm{~kg} / \mathrm{ha}$, mientras que para los estanques inorgánico y orgánico fue de $1310.14 \mathrm{~kg} / \mathrm{ha}$ y $1396.14 \mathrm{~kg} / \mathrm{ha}$ respectivamente, lo que indica un efecto positivo de la fertilización en la producción de tenca.

El hecho de "abonar"constituye una estrategia favorable en la producción de tenca, sin embargo, la decisión sobre el tipo de abono a emplear está subordinada fundamentalmente a razones de interés para la empresa, como son, factores económicos (disponibilidad en el mercado, precio, cantidad necesaria...), de manejo (transformación previa, aplicación...) y ambientales (limpieza, sustancias húmicas no controladas...), más que a favorecer el desarrollo de un determinado grupo zooplanctónico, que a su vez pudiera tener un efecto positivo sobre la producción de tenca.

\section{BIBLIOGRAFÍA}

ALONSO, M. 1985. Las lagunas de la España Peninsular: Taxonomía, ecología y distribución de 
los Cladóceros. Tesis Doctoral., Universidad de Barcelona. $795 \mathrm{pp}$.

ALONSO, M. 1996. Fauna Ibérica. Vol. 7: Crustacea. Branchiopoda. Museo Nacional de Ciencias Naturales (CSIC). Madrid. 486 pp.

APHA 1985. Standard Methods for the Examination of Water and Waste Water. 16th ed. Washington D.C. $1201 \mathrm{pp}$.

ARMENGOL, J. 1978. Los crustáceos del plancton de los embalses españoles. Oecologia Aquatica, 3 : 3-96.

ARORA, J. \& N. K. MEHRA, 2003. Seasonal dynamics of rotifers in relation to physical and chemical conditions of the river Yamuna (Delhi), India. Hydrobiologia, 491: 101-109.

BERZINS, B. \& B. PEJLER. 1987. Rotifer occurrence in relation to $\mathrm{pH}$. Hydrobiologia, 147: 107116.

BERZINS, B. \& B. PEJLER. 1989. Rotifer occurrence and trophic degree. Hydrobiologia, 182: 171-180.

BOTTRELL, H. H., A. DUNCAN. Z. M. GLIWICZ, E. GRYGIERIK, A. HERZIG, A. HILLBRICHTILKOWSKA, H. KURASAWA, P. LARSON \& T. WEGLENSKA. 1976. A review of some problems in zooplankton production studies. Norwegian Journal of Zoology, 24: 419-456.

BOYD, C. E. 1982. Water Quality Management for Pond Fish Culture. Elsevier Scientific Publishing Company, New York, 318 pp.

BRAIONI, M. G. \& D. GELMINI. 1983. Guide per il reconoscimento delle specie animali delle acque interne etaliane. Rotiferi Monogononti. Consiglio Nazionalie delle Ricerche. 180 pp.

BRETT, M. T. \& C. R. GOLDMAN. 1997. Consumer versus resource control in freshwater pelagic food webs. Science, 275: 384-386.

CARPENTER, S. R., J. F. KITCHELL, J. R. HODGSON, P. A. COCHRAN, J. J. ELSER, M. M. ELSER, D. M. LODGE, D. KRETCHMER, X. HE \& C. N. VON ENDE. 1987. Regulation of lake primary productivity by food web structure. Ecology, 68: 1863-1876.

CHAKRABARTI, R. \& B. B. JANA. 1992. Diversity and abundance of plankton as indexes of management strategies of common carp culture. Archiv Hydrobiol., 125 (4): 499-509.

CHRISTOPHER, F. S. \& H. R. ALLISON. 2003. Seasonal succession in fishless ponds: effects of enrichment and invertebrate predators on zooplankton community structure. Hydrobiologia, 490: $125-134$.

DEVETTER, M. 1998. Influence of enviromental factors on the rotifer assemblages in an artificial lake. Hydrobiologia, 387/388: 171-178.

DOERING, P. H., C. A. OVIATT, L. L. BEATTY, V. F. BANZON, R. RICE, S. P. KELLY, B. K. SULLIVAN \& J. B. FRITHSEN. 1989. Structure and function in a model coastal system: silicon, the benthos and eutrophication. Mar. Ecol Prog Ser., 52: 287-299.

DUGGAN, I. C., J. D. GREEN \& K. THOMASSON. 2001. Do rotifers have potencial as bioinficators of lake trophic state? Verh. Internat. Verein. Limnol., 27: 3497-3502.

DUMONT, J. H., I. VAN DE VELDE \& S. DUMONT. 1975. The Dry Weight Estimate of Biomasa in a Selection of Cladocera, Copepoda and Rotifera from the plankton, periphyton and benthos of continental waters. Oecologia, 19: 75-97.

DUSSART, B. 1967. Les copepodes des eaux continentales d'Europe occidental. Tome I : Calanö̈des et Harpacticoïdes. N. Boubée \& Cie. Paris. 500 pp.

DUSSART, B. 1969. Les copepodes des eaux continentales d'Europe occidental. Tome II: Cyclopoïdes et biologie. N. Boubée \& Cie. Paris. 292 pp.

EJSMONT-KARABIN, J. 1995. Rotifer occurrence in relation toa ge, depth and trophic state of quarry lakes. Hydrobiologia, 313/314: 21-28.

FERNÁNDEZ-ALÁEZ, M, C. FERNÁNDEZALÁEZ, E. BÉCARES, M. VALENTIN, J. GOMÁ \& P. CASTRILLO. 2004. A two-years experimental study on nutrient and predator influences on food web constituents in a shallow lake of north-west Spain. Freswat. Biol., 49: 1574-1592.

FULTON, R. S. 1984. Predation, production and the organization of an estuarine copepod community. J. Plankton Res., 6: 399-415.

HANSSON, L. A. 1992. The role of food chain composition and nutrient availability in shaping algal biomass development. Ecology, 73: 241-247.

HORPPILA, J., H. PELTONEN, T, MALINEN, E, LUOKKANEN \& T. KAIRESALO. 1998. Topdown or bottom-up effects of fish: issues of concern in biomanipulation of lakes. Restoration Ecology, 6: 20-28.

HORSTED, S. J., T. G. NIELSEN, B. RIEMANN, J. POCKSTEEN \& P. K. BJORNEN. 1998 Regulation of zooplankton by suspension-feeding 
bibalves and fish in estuarine enclosures. Mar Ecol Prog Ser., 48: 217-224.

HUTCHINSON, G. E. 1967. A treatise on Limnology. Vol. II: Introduction to Lake Biology and Limnoplancton. Wiley. New York. 1115 pp.

IGER, Y., M. ABRAHAM, A. DOTAN, B. FATTAL, \& E. RAHAMIM. 1988. Cellular responses in the skin of carp maintained in organically fertilized water. J. Fish Biol., 33(5): 711-720.

JEPPESEN, E., J. P. JENSEN, M. SØNDERGAARD, T. LAURIDSEN, L. J. PEDERSEN \& L. JENSEN. 1997. Top-down control in freshwater lakes: the role of nutrient state submerged macrophytes and water depth. Hydrobiologia, 342/343: 151-164.

JEPPESEN, E., T. LAURIDSEN, T. KAIRESALO \& M. PERROW. 1998. Impact of submerged macrophytes on fish-zooplankton interactions in lakes. In: The Structuring Role of Submerged Macrophytes in Lakes. E. Jeppesen, Ma. Søndergaard, Mo. Søndergaard \& K. Christoffersen (eds): $177-$ 189. Springer-Verlag, New York.

KAIRESALO, T., S. LAINE, E. LUOKKANEN, T. MALINEN \& J. KETO. 1999. Direct and indirect mechanisms behind successful biomanipulation. Hydrobiologia, 395/396: 99-106.

KAUSHIK, S. \& D. N. SAKSENA, 1995. Trophic status and rotifer fauna of certain water bodies in Central India. J. Environ. Biol., 16: 283-291.

KELLER, A. A., P. H. DOERING, S. P. KELLY \& B. K. SULLIVAN. 1990 Growth of juveniles atlantic menhaden Brevoortia tyrannus (Pisces:Clupeidae) Limnol Oceanogr., 35: 109-122.

KENNEDY, M. \& P. FITZMAURICE. 1970. The biology of the Tench tinca tinca (L.) in Irish Waters. Inland Fisheries Trust, Dublin. Proc. R.I.A., vol 69, sect B.

KNUD-HANSEN, C. F. 1998. Pond fertilization: ecological approach and practical applications. Pond dynMICS/Aquaculture collaborative research support program. Oregon State University, Corvallis, Or. 125 pp.

LEIBOLD, M. A., J. M. CHASE, J. B. SHURIN \& A. L. DOWNING. 1997. Species turnover and the regulation of trophic structure. Ann. Rev. Ecol.
Syst., 28: 467-494.

McQUENN, P. J., J. R. POST \& E. L. MILLS. 1986. Trophic relationships in freshwater pelagic ecosystems. Can. J. Fish. Aquat. Sci., 43: 1571-1581.

MARGARITORA, F. G. 1985. Fauna d'Italia. Cladocera. Edizioni Calderini. Bologna. 389 pp.

MAZUMDER, A. 1994. Patterns of algal biomass in dominant odd- vs. even-link lake ecosystems. Ecology, 75: 1141-1149.

OKONJI V. A. \& A. OBI. 1999. Effects of three fertilizer treatments on zooplankton productivity in plastic tanks. Indian Journal of Animal Sciences, 69(5): 360-363.

PATALAS, K. 1972. Crustacean plankton and the eutrophication of St Laurence Great Lakes. J. Fish. Res. Board Can., 29: 1451-1462.

PERSSON, L., G. ANDERSSON, S. F. HAMRIN \& L. JOHANSSON. 1988. Predator regulation and primary production along the productivity gradient of temperate lake ecosystems. In: Complex Interactions in Lake Communities S.R. Carpenter (ed.): 45-65. Springer-Verlag, Berlín.

PERSSON, L., S. DIEHL, L. JOHANSSON, G. ANDERSON \& S. F. HAMRIN. 1992. Trophic interactions in temperate lake ecosystems: a test of food chain theory. Am. Nat., 140: 59-87.

SARNELLE, O. 1992. Nutrient enrichment and grazer effects on phytoplankton in lakes. Ecology, 73: 551-560.

SIEGFRIED, C. A., J. A. BLOOMFIELD \& J. W. SUTHERLAND. 1989. Planktonic rotifer community structure in Adironack, New York, U.S.A lakes in relation to acidity, trophic status and related water quality characteristics. Hydrobiologia, 175: 33-48.

STEINER, F. \& A. H. ROY. 2003. Sesasonal succession in fishless ponds: effects of enrichment and invertebrate predators on zooplankton community structure. Hidrobiología, 490: 125-134.

VELASCO J. L. 1990. Lista faunística y bibliográfica de los Rotíferos (Rotatoria) de la Península Ibérica e Islas Baleares y Canarias. Asociación Española de Limnología. Museo Nacional de Ciencias Naturales. Madrid. 195 pp. 